\title{
Pre- and post-transition behavior of shear-thickening fluids in oscillating shear
}

\author{
Christian Fischer - Christopher J. G. Plummer • \\ Véronique Michaud • Pierre-Etienne Bourban • \\ Jan-Anders E. Månson
}

Received: 31 May 2006 / Accepted: 2 May 2007 / Published online: 20 May 2007

(C) Springer-Verlag 2007

\begin{abstract}
The dynamic shear-thickening behavior of concentrated colloidal suspensions of fumed silica in polypropylene glycol has been investigated. Dynamic frequency sweeps showed that, for any given solids concentration, the complex viscosity at different imposed strain amplitudes followed a unique power-law-type behavior up to the onset of strain thickening. Moreover, similar behavior was also observed in the post-transition state, i.e., the viscosities again superimposed at frequencies beyond the transition frequency. In an attempt to develop a parametric description of this behavior, both the Delaware-Rutgers rule and the concept of a critical shear stress for the onset of shear thickening in steady-state experiments were considered. However, neither approach could account for the observed trends over the entire range of strains and frequency investigated. Plots of the critical shear strains for the onset and the end-point of the transition as a function of frequency were, therefore, used to describe the state of the suspensions for an arbitrary combination of strain and frequency. Finally, Fourier transform (FT) rheology was used to evaluate the extent of non-linearity in the response of the suspensions to dynamic shear, and it was shown that the observed behavior was not significantly influenced by wall slip at the tool-specimen interface.
\end{abstract}

This paper was presented at the third Annual Rheology Conference, AERC 2006, April 27-29, 2006, Crete, Greece.

C. Fischer · C. J. G. Plummer · V. Michaud - P.-E. Bourban •

J.-A. E. Månson $(\bowtie)$

Laboratoire de Technologie des Composites et Polymères (LTC),

Ecole Polytechnique Fédérale de Lausanne (EPFL),

CH-1015 Lausanne, Switzerland

e-mail: jan-anders.manson@epfl.ch
Keywords Shear thickening $\cdot$ LAOS $\cdot$ Silica suspension . FT rheology
Abbreviations
STF shear-thickening fluid
FT Fourier transform
ODT order-to-disorder transition

\section{Introduction}

Research into shear-thickening fluids (STFs) has accelerated sharply over the last few years. Initially, interest in shear thickening derived mainly from its association with damage to processing equipment and dramatic changes in suspension microstructure, e.g., particle aggregation, which is known to compromise a range of properties. However, it was soon recognized that the highly nonlinear behavior of STFs could be exploited in the design of damping devices and machine mounts, (Helber and Dockner 1990; Laun et al. 1991) and more recently, there has been considerable interest in STFs in the context of body armor, owing to their ability to absorb high amounts of energy when impacted with high velocity projectiles (Lee et al. 2003).

At low but increasing shear rates, STFs generally undergo weak shear thinning, which may be described by a power-law-type relationship, but once a critical shear rate, $\dot{\gamma}_{c}$, is reached, the viscosity, $\eta$, increases sharply. In steady-state measurements, the shear-thickening transition in STFs is typically complete within about one decade of shear rate, $\dot{\gamma}$, of its onset (Barnes 1989). Beyond the end point of the transition, denoted $\dot{\gamma}_{m}$, three different phenomena may occur: (1) Fracture of the specimen may intervene owing to the high $\eta$, (2) $\eta$ may become 
approximately independent of $\dot{\gamma}$, or (3) $\eta$ may decrease with increasing $\dot{\gamma}$ (Hoffmann 1972).

Although there is now a substantial volume of literature in the field, the origins of shear thickening in colloidal suspensions were not clearly understood until recently. Early experiments performed by Hoffman (Hoffmann 1972, 1974) suggested it to be a consequence of an order-todisorder transition (ODT). Based on this idea, Boersma et al. (1990) proposed that the critical shear rate for thickening could be obtained by considering the balance between electrostatic and shear forces acting on pairs of particles. Laun and Bung (1992) were the first to question the validity of this approach, and various studies have since confirmed shear thickening to be due to "hydrodynamic clustering" rather than an ODT (Bossis and Brady 1989; Bender and Wagner 1996; Foss and Brady 2000; Maranzano and Wagner 2001a, b; Lee and Wagner 2003). For example,
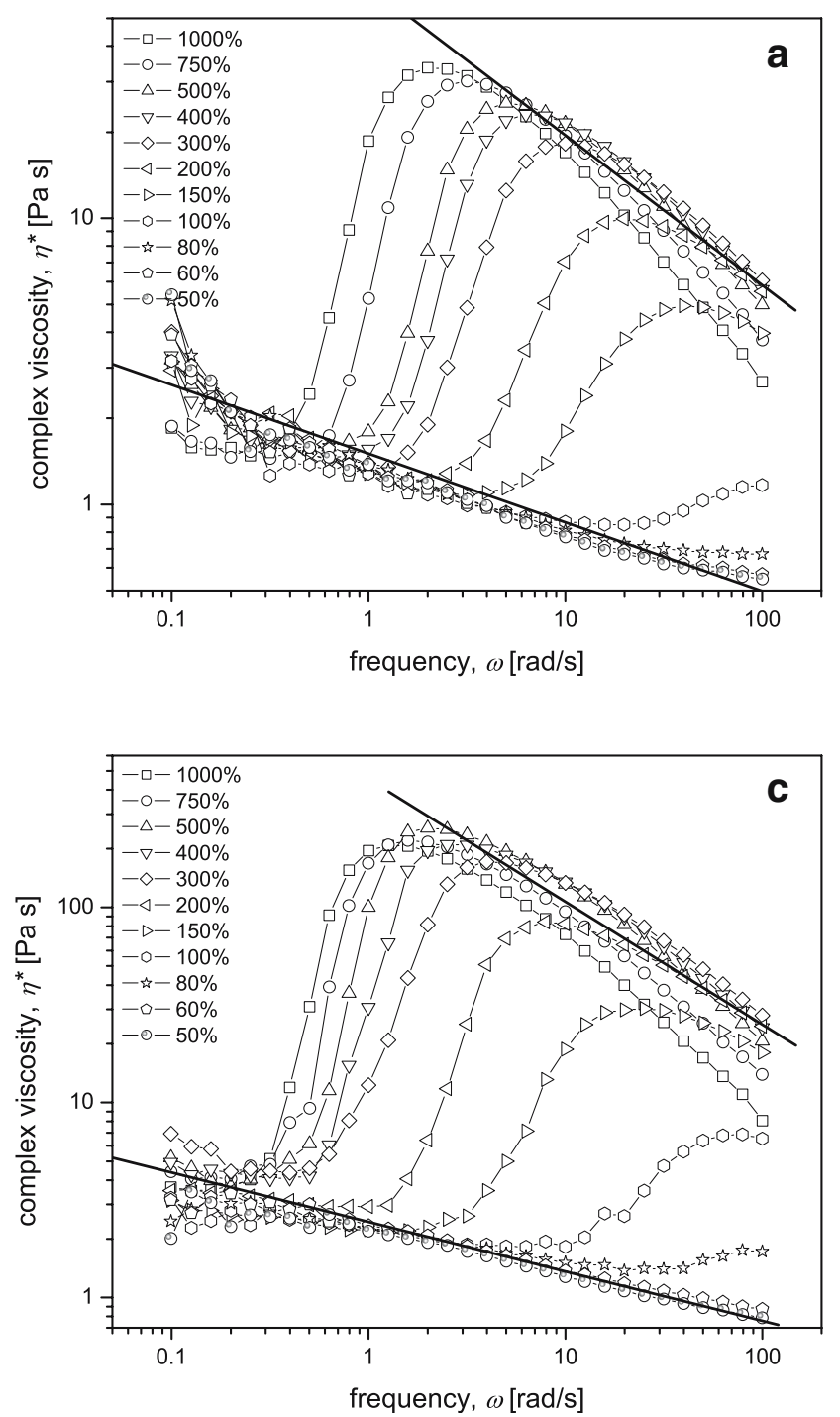

Bossis and Brady (1989) simulated the viscosity of a suspension of spherical Brownian particles by Stokesian dynamics as a function of the Péclet number. According to their results, which were in good correlation with the experiments, the suspension shear thickens, owing to the formation of large clusters at very high Péclet numbers. These hydrodynamic clusters are composed of compact groups of particles formed as shear forces drive them into contact, and short-range lubrication forces dominate the observed viscosity increase (Bender and Wagner 1996). A range of parameters control this behavior, among the most important being the particle size distribution, particle content, particle shape (Clarke 1967), particle-particle interactions, and the viscosity of the continuous phase (Barnes 1989). The cumulative effect of these factors determines the extent of shear thickening and the sharpness of the transition, as well as the post-transition response.
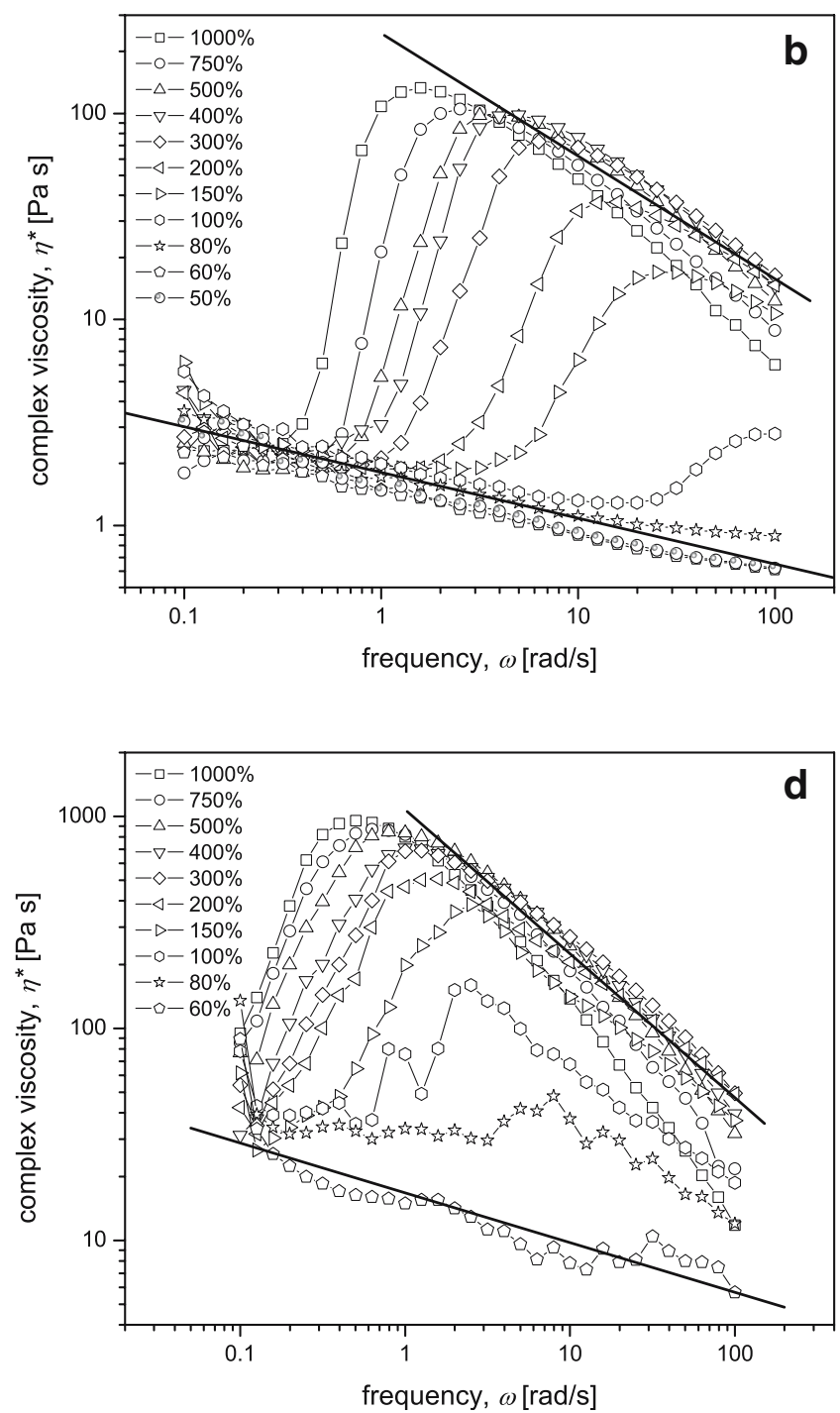

Fig. 1 Dynamic frequency sweeps at set $\gamma_{0}$ from 50 to $1,000 \%$ for a $10 \% w / w$, b $12.5 \% w / w$, c $15 \% w / w$, and d $17.5 \% w / w$ silica-PPG. The straight lines are exponential fits to the pre- and post-transition behavior 

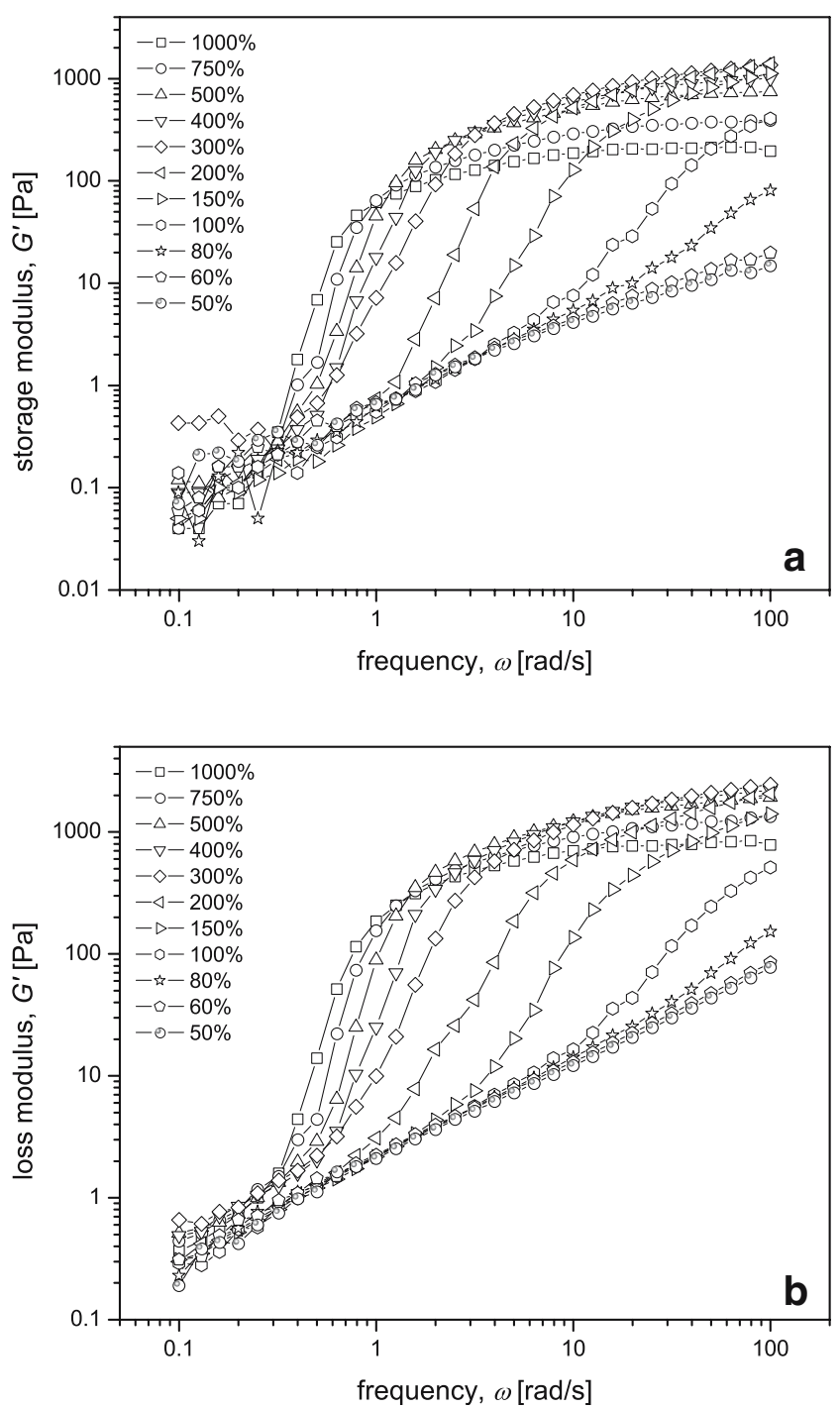

Fig. 2 a Storage modulus and b loss modulus of the $15 \% w / w$ suspension under dynamic frequency sweeps at set $\gamma_{0}$ from 50 to $1,000 \%$

There has been comparatively little research into the dynamic properties of STFs. Laun et al. (1991) reported the critical strain amplitude for dynamic shear thickening, $\gamma_{c}$, at fixed angular frequency, $\omega$, of a polymer latex dispersion to decrease with increasing $\omega$, but eventually, to reach a plateau at the highest $\omega$. Following Boersma et al. (1992) who investigated monodisperse silica particles suspended in a mixture of glycerol and water, they interpreted the low $\omega$ behavior in terms of the steady-state shear response, i.e., $\dot{\gamma}_{c}^{\text {dynamic }}=\gamma_{c} \omega \approx \gamma_{c}$ steady . The nature of the high $\omega$ behavior, however, remains less clear. Some authors have suggested that a minimum strain amplitude is necessary for shear thickening, even at the highest $\omega$ (Laun et al. 1991; Raghavan and Khan 1997), whereas others have observed a similar frequency dependence to that at low $\omega$ (Boersma et al. 1992; Mewis and Biebaut 2001).
The current investigation has been carried out within the framework of a study aimed at integrating STFs into a composite sandwich structure to induce changes in its dynamic properties under specific conditions. We have shown previously that such a model structure can undergo an increase in rigidity and damping simultaneously under high amplitude deformation (Fischer et al. 2006a), behavior that is of particular interest for sports engineering, e.g., alpine skis (Fischer et al. 2006b). The focus in this study is on the shear-thickening behavior of highly concentrated suspensions of fumed silica in polypropylene glycol (PPG) under large amplitude oscillatory shear (LAOS). A working description is sought for the behavior of the STF for any strain and frequency combination that can be used in conjunction with structural models. Special attention has, therefore, been paid to the response of the STF before and after the transition, and Fourier transform (FT) rheology has been used to investigate whether it is necessary to consider slip at the tool-sample interface in interpreting the rheological data.

\section{Materials and methods}

The STF was based on hydrophilic fumed silica (Aerosil 200, from Degussa), which has a primary (spherical) particle size of $12 \mathrm{~nm}$ and a specific surface area of approximately $200 \mathrm{~m}^{2} / \mathrm{g}$. The primary particles are irreversibly bonded to form large aggregates that, according to the supplier, cannot be disrupted by shear. The carrier fluid was polypropylene glycol $\left(\mathrm{H}\left[\mathrm{OCH}\left(\mathrm{CH}_{3}\right) \mathrm{CH}_{2}\right]_{\mathrm{n}} \mathrm{OH}\right.$ (from Sigma-Aldrich) with an average molar mass of $1,000 \mathrm{~g} / \mathrm{mol}$. In each case, the carrier fluid was added to the powder,

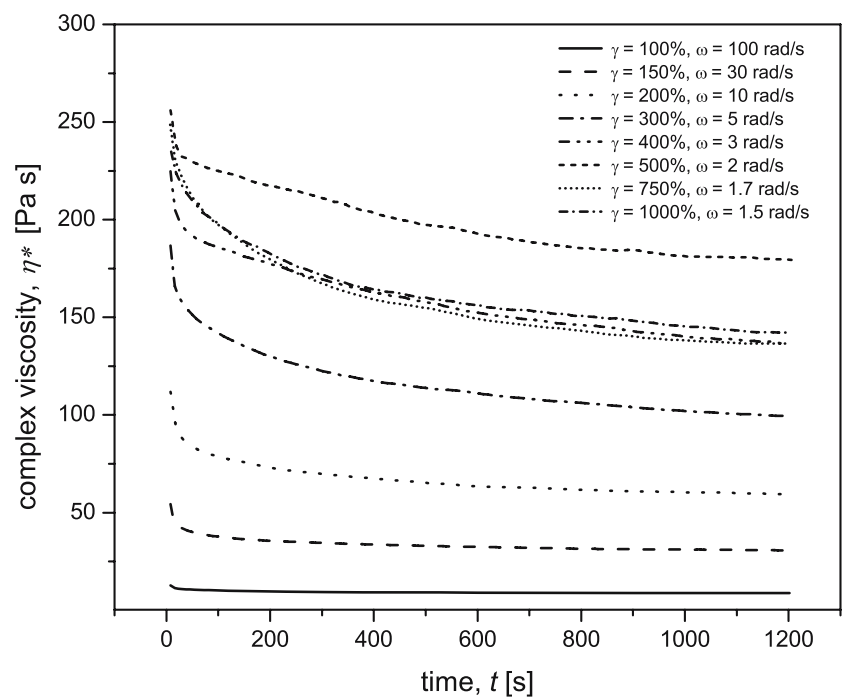

Fig. 3 Time dependence of $\eta^{*}$ for the $15 \% w / w$ suspension using strain-frequency combinations corresponding to the completion of the transitions in Fig. 1c 
and a blender was used to mechanically mix the two components for $5 \mathrm{~min}$. The resulting suspensions were then placed in a vacuum chamber for several hours to eliminate any bubbles. The concentrations tested were $10,12.5,15$, and $17.5 \% \mathrm{w} / \mathrm{w}$.

Rheological measurements were performed on a straincontrolled Rheometrics Scientific ARES rheometer. Steadystate strain-rate sweeps, dynamic frequency sweeps, and dynamic strain sweeps were carried out using $25-\mathrm{mm}$ diameter parallel plates with a gap of $400 \mu \mathrm{m}$, and to check for instrumental artifacts, selected measurements were also carried out using a $25-\mathrm{mm}$ diameter cone-plate tool with a cone angle of $0.1 \mathrm{rad}$. To minimize wall slip at the specimen-tool interfaces, the surfaces of the plates were covered with emery paper. The 10, 12.5, and $15 \% \mathrm{w} / \mathrm{w}$ specimens were conditioned by shearing at $1 \mathrm{~s}^{-1}$ for $60 \mathrm{~s}$ before each measurement. In the case of the $17.5 \% \mathrm{w} / \mathrm{w}$ specimens, a pre-shear of $0.1 \mathrm{~s}^{-1}$ was used so as to avoid initiating the thickening transition.

For the FT rheology, the ARES was equipped with an electromagnetically compensated torque transducer (2KFRTN1). The data were externally digitized using a 16-bit analogue-to-digital converter (ADC) card (PCI-MIO16XE; National Instruments) that allowed the measurement and averaging (over-sampling) of the shear strain and the shear torque simultaneously. LabView 5.1 routines (National Instruments) were used to acquire and handle raw time data and to apply the FT analysis. Over-sampling permitted a fivefold decrease in the minimum detectable stress and significant improvements in the signal-to-noise ratio $(S / N)$. More detailed information on the experimental set-up is given elsewhere (Wilhelm 2002; Schmidt et al. 2005).
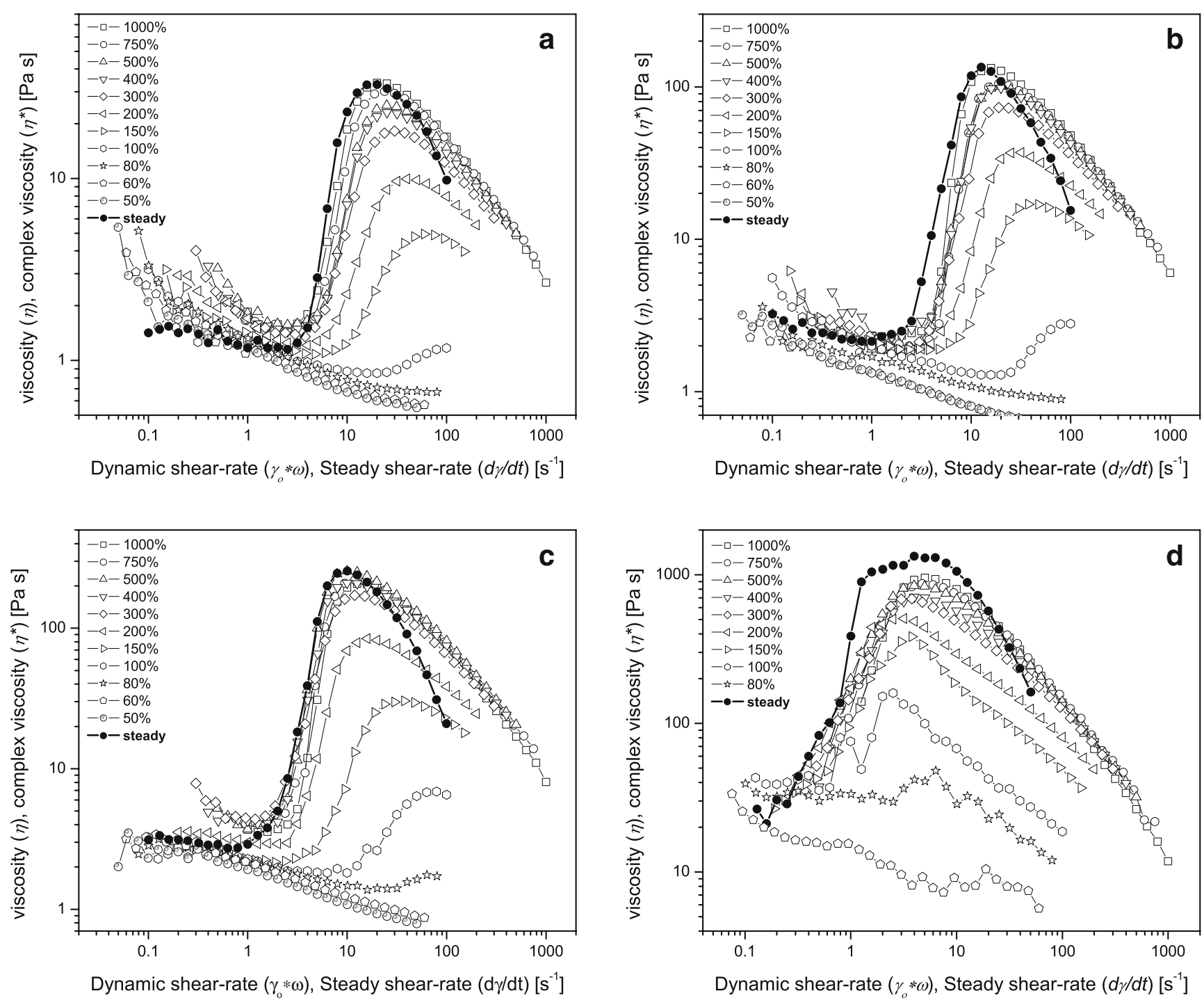

Fig. 4 Delaware-Rutgers rule applied to a $10 \% w / w$, b $12.5 \% w / w$, c $15 \% w / w$, and d $17.5 \% w / w$ silica-PPG suspensions (data from dynamic frequency sweeps for $0.1<\omega<100 \mathrm{rad} / \mathrm{s}$ at various $\gamma_{0}$ and steady strain rate sweeps) 


\section{Results and discussion}

Pre- and post-transition behavior

Figure 1 shows the complex viscosity, $\eta^{*}$, as a function of $\omega$ for the different fumed silica-PPG suspension at different set strains, $\gamma_{0}$. For any given solid content, the transition initiated at relatively low $\omega$ when $\gamma_{0}$ was high and the critical value of $\omega$ increased monotonically with decreasing $\gamma_{0}$. In the range of $\omega$ below the transition, all the specimens showed approximately the same power-law behavior. The curves also superposed beyond the transition, with the exception of those for $\gamma_{0}=750$ and $1,000 \%$. However, the deviations of these latter from the general trend could be attributed to cohesive failure of the specimens. These deviations were also apparent in the data for the storage modulus, $G^{\prime}$, and the loss modulus, $G^{\prime \prime}$, examples of which are given in Fig. 2 for the $15 \% w / w$ suspension. The $17.5 \%$ $w / w$ suspension was particularly difficult to handle, owing to its very high viscosity and its very early shear-thickening transition. The data for this concentration, therefore, showed a relatively high degree of scatter.

Systems frequently investigated for their shear-thickening behavior often show a far more abrupt transition, and their post-transition behavior cannot be characterized owing to technical difficulties associated with the very high viscosities (Maranzano and Wagner 2001a,b; Lee and Wagner 2003; Egres and Wagner 2005). A rare example of post-transition data is provided by Laun et al. (1991) and is briefly discussed in the review by Barnes (1989), where the possibility of post-transition shear thinning is discussed. In the present case, inspection of Fig. 1 indicates that, for any given suspension, either in the low viscosity pretransition state or in the post-transition thickened state, $\eta^{*}$ $(\omega)$ followed a unique power law. Moreover, $\eta^{*}$ decreased more rapidly beyond the transition than at low frequencies, implying that the shear-thickening transition may disappear at sufficiently high frequencies. Owing to the limitations of the equipment, this could not be verified experimentally.

Investigations of the time dependence of $\eta^{*}$ for the $15 \%$ $w / w$ suspension using strain-frequency combinations corresponding to the completion of the transitions in Fig. 1c, i.e., the thickened state, indicated a slight decrease with time (Fig. 3). This phenomenon was apparently more pronounced as the set strain increased. The large decreases in $\eta^{*}$ in the case of the curves for 500 and $750 \%$ strain were again thought to reflect cohesive failure of the specimens. According to the supplier, the relatively low shear stresses reached under the present test conditions were not sufficient to disrupt the fumed silica agglomerates. The higher shear stresses beyond the thickening transition may nevertheless lead to a rearrangement of the spatial dispersion of these agglomerates, resulting in the observed decrease in $\eta^{*}$. The evolution of the state of the suspension has not so far been investigated for longer time scales. However, the decrease in $\eta^{*}$ with time during the first seconds after the application of the strain (the time domain corresponding to the frequency sweeps shown, for example, in Fig. 1) was significantly weaker than the decrease in $\eta^{*}$ with $\omega$ associated with the post-transition behavior of the suspension (Fig. 1c). Hence, this phenomenon did not significantly affect the posttransition behavior discussed earlier. A fresh sample was nevertheless used for each measurement as a precaution.

Determination of the critical shear strain

Figure 4 shows the data in Fig. 1 rescaled to investigate the applicability of the Delaware-Rutgers rule:

$\eta^{*}\left(\gamma_{0} \omega\right)=\eta(\dot{\gamma})$
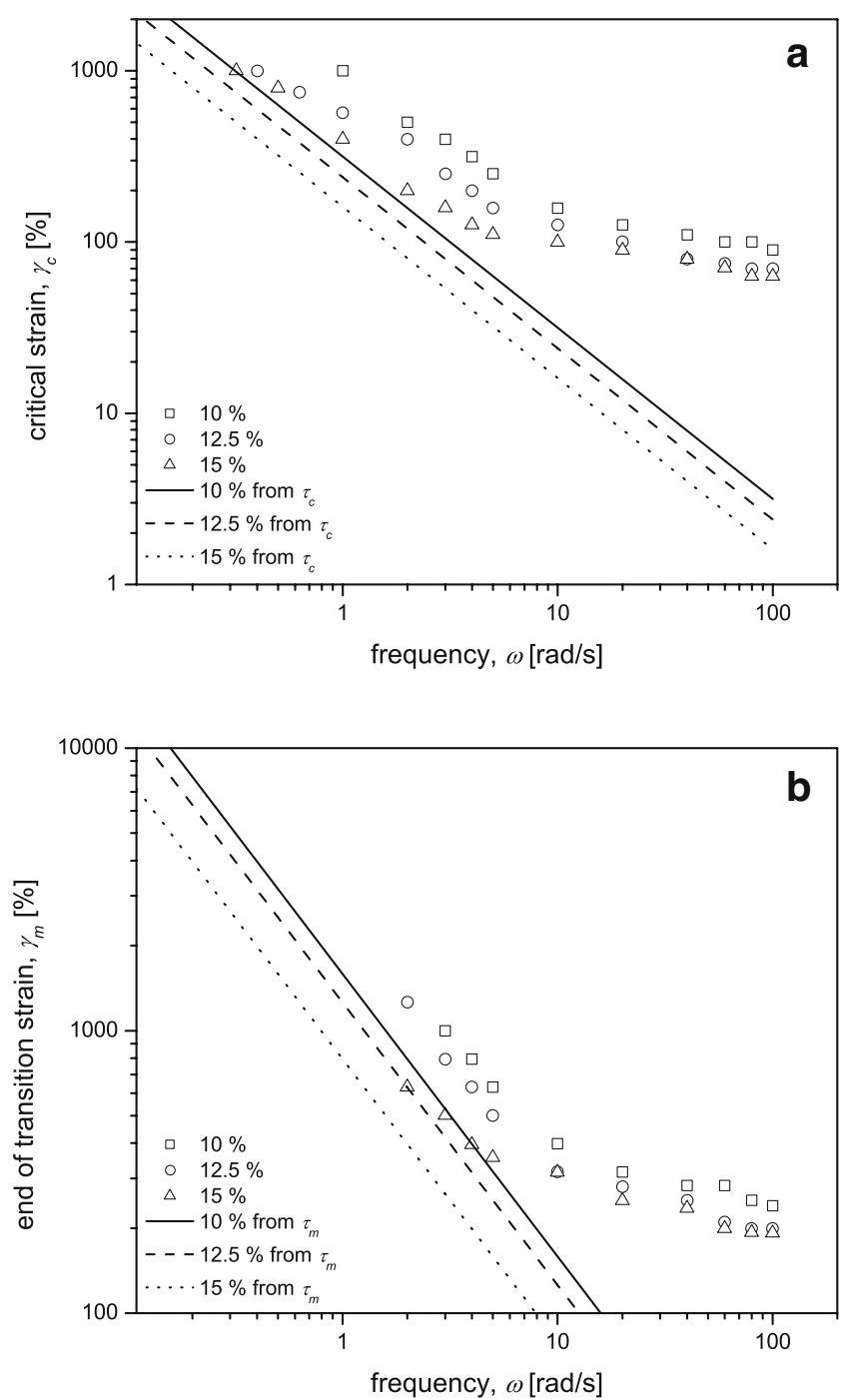

Fig. 5 Comparison between experimental results (open symbols) and predictions from Eq. 2 (straight lines) for $\mathbf{a} \gamma_{\mathrm{c}}$ and $\mathbf{b} \gamma_{\mathrm{m}}$ for $10 \% \mathrm{w} / \mathrm{w}$, $12.5 \% \mathrm{w} / \mathrm{w}$, and $15 \% \mathrm{w} / \mathrm{w}$ silica-PPG suspensions 
which is often used to establish correlations between $\eta^{*}$ (obtained from a frequency sweep at a constant strain amplitude $\gamma_{0}$ ) and the steady-state shear viscosity $\eta$ (Doraiswamy et al. 1991). The curves for high $\gamma_{0}$ transposed relatively well onto the steady-state data in the transition and post-transition regimes, but the curves for $\gamma_{0}<300 \%$ did not. This was consistent with the results of Raghavan and Khan (1997) who also observed superposition at high strain-amplitudes only $\left(\gamma_{0}>500 \%\right)$ when investigating similar materials with lower solids contents. Also consistent with the results of Raghavan and Khan (1997) was the observation that the steady-state curve was always displaced to low frequencies with respect to the rescaled dynamic curves. However, contrary to those results and also to those of Lee and Wagner (2003), who investigated monodisperse suspensions, in the present case, the superposition for high strain amplitudes was poor in the pre-transition regime.
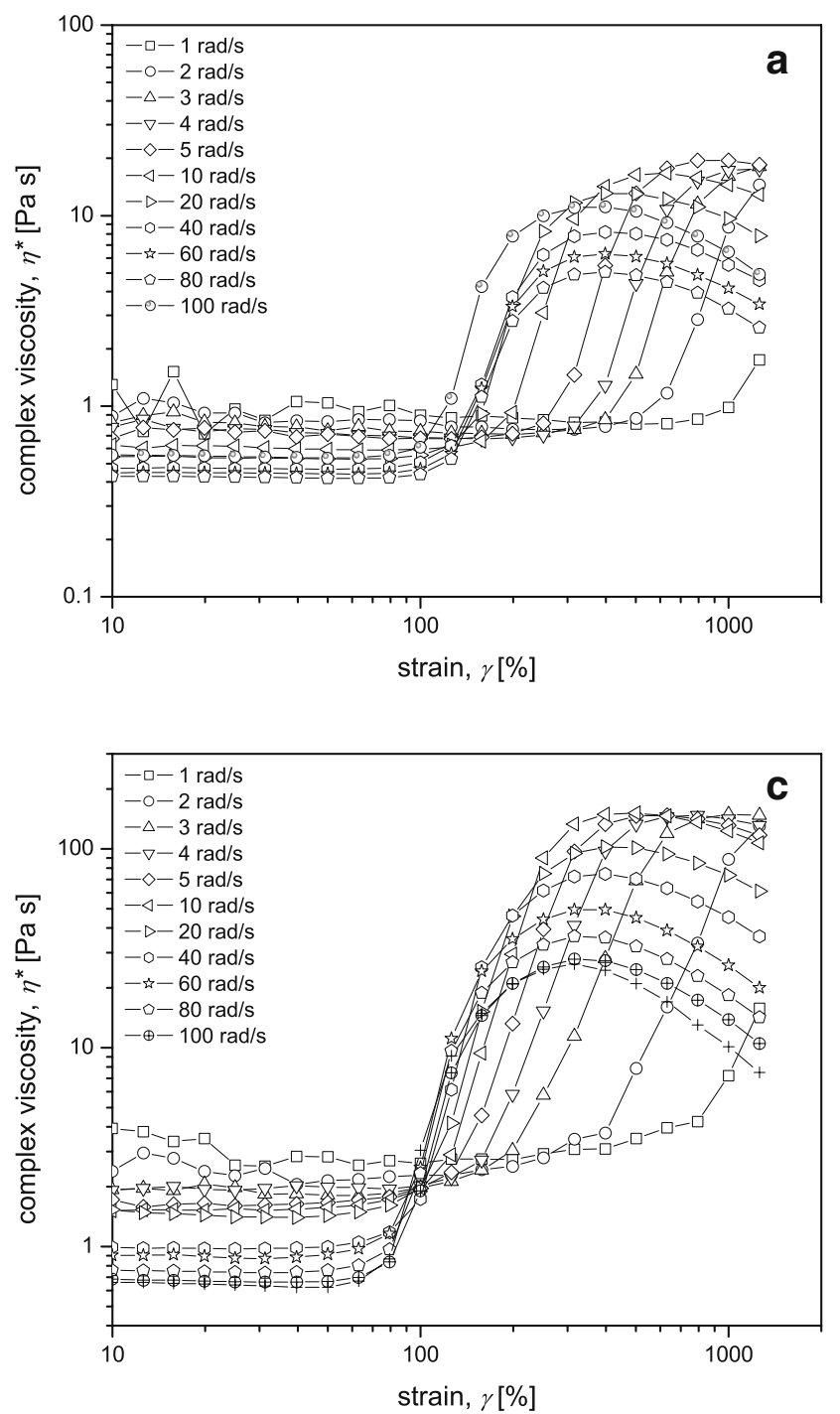

Recent publications on shear-thickening colloidal suspensions have suggested shear thickening to be a stresscontrolled phenomenon (Bender and Wagner 1996; Maranzano and Wagner 2001a, b; Krishnamurthy et al. 2005). Based on this principle, Lee and Wagner proposed that shear thickening should also be governed by the critical shear stress, $\tau_{\mathrm{c}}$, in dynamic shear. Thus, assuming that shear thickening occurs at $\tau>\tau_{\mathrm{c}}$, one may write the corresponding strain as (Lee and Wagner 2003)

$\gamma_{c} \approx \frac{\tau_{c}}{\eta_{c} \omega}$

where $\eta_{\mathrm{c}}$ is the shear viscosity at the thickening onset, both of which may be determined from the steady-state shear data. Equation 2 accounted well for Lee and Wagner's data when emery paper was present on the tool surfaces, and they showed that possible deviations from it were due to wall slip at the tool-sample interface. They, thus, concluded that the
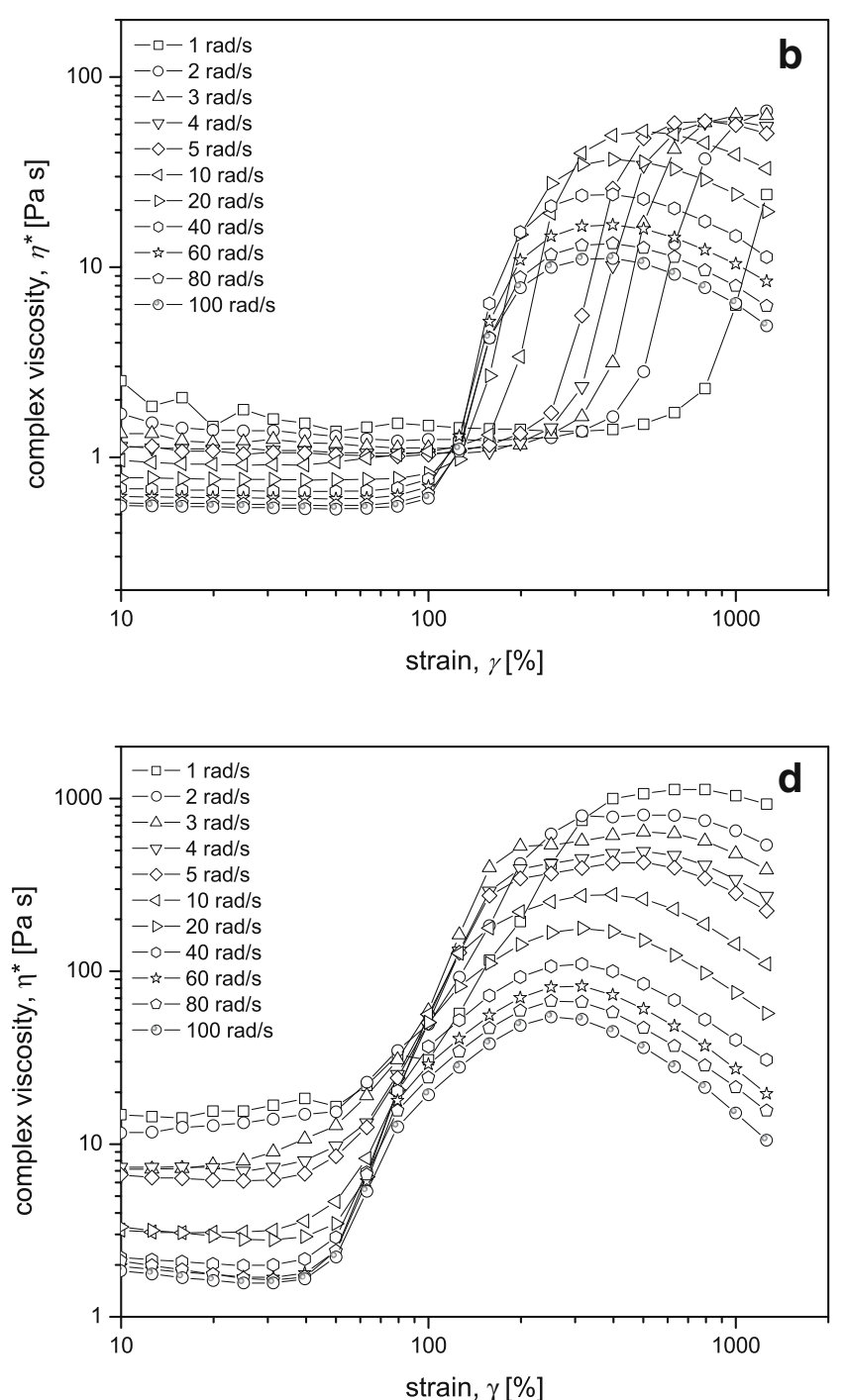

Fig. 6 Dynamic strain sweeps at fixed $\omega$ from 1 to $100 \mathrm{rad} / \mathrm{s}$ for a $10 \% w / w$, b $12.5 \% w / w$, c $15 \% w / w$, and d $17.5 \%$ w $/ w$ silica-PPG suspensions 
use of emery paper was crucial for this type of measurement. In the present case, however, $\gamma_{\mathrm{c}}$ from the dynamic measurements could not simply be described by $\tau_{\mathrm{c}}$ (Fig. 5), as the calculated $\gamma_{\mathrm{c}}$ under-predicted the response at low $\omega$, and even stronger deviations occurred as $\omega$ increased. The same trends were observed when applying Eq. 2 to the strain corresponding to the completion of the transition, $\gamma_{\mathrm{m}}$ (Fig. 5b).

In conclusion, then, the state of the suspension could be adequately described by neither $\dot{\gamma}$, nor $\tau_{\mathrm{c}}$, i.e., $\dot{\gamma}_{c}$ varied with varying $\omega$. Therefore, to characterize the state and the viscosity of a suspension subjected to a certain combination of strain and frequency, the critical strains, $\gamma_{\mathrm{c}}$ and $\gamma_{\mathrm{m}}$, were plotted as a function of $\omega$ for the different suspensions, as shown in Fig. 7. The data in Fig. 7 were compiled from both dynamic strain sweeps at different $\omega$ (Fig. 6), and frequency sweeps at different $\gamma_{o}$ (Fig. 1). For comparison, the dashed lines represent $\gamma_{\mathrm{c}}$ values predicted by applying the Delaware-Rutgers rule to the steady shear data (cf. Fig. 3), showing poor agreement with the experimental data at high $\omega$. A plateau in the experimental $\gamma_{\mathrm{c}}$ at high $\omega$ was also reported by Raghavan and Khan (1997). For any given concentration, such plots could be used to predict whether the STF was in the pre-transition, the transition, or the posttransition shear-thickened state. Power law fits (cf. Fig. 1) could then be used to estimate the pre- and post-transition values of $\eta^{*}$ for any $\gamma_{0}$ and $\omega$.

\section{Fourier transform rheology}

High $\omega$ plateaux such as seen in the $\gamma_{\mathrm{c}}$ and $\gamma_{\mathrm{m}}$ data in Fig. 7 have been suggested to relate to slip at the sample-tool interface (Lee and Wagner 2003), although the same authors suggested that the effect could be avoided by
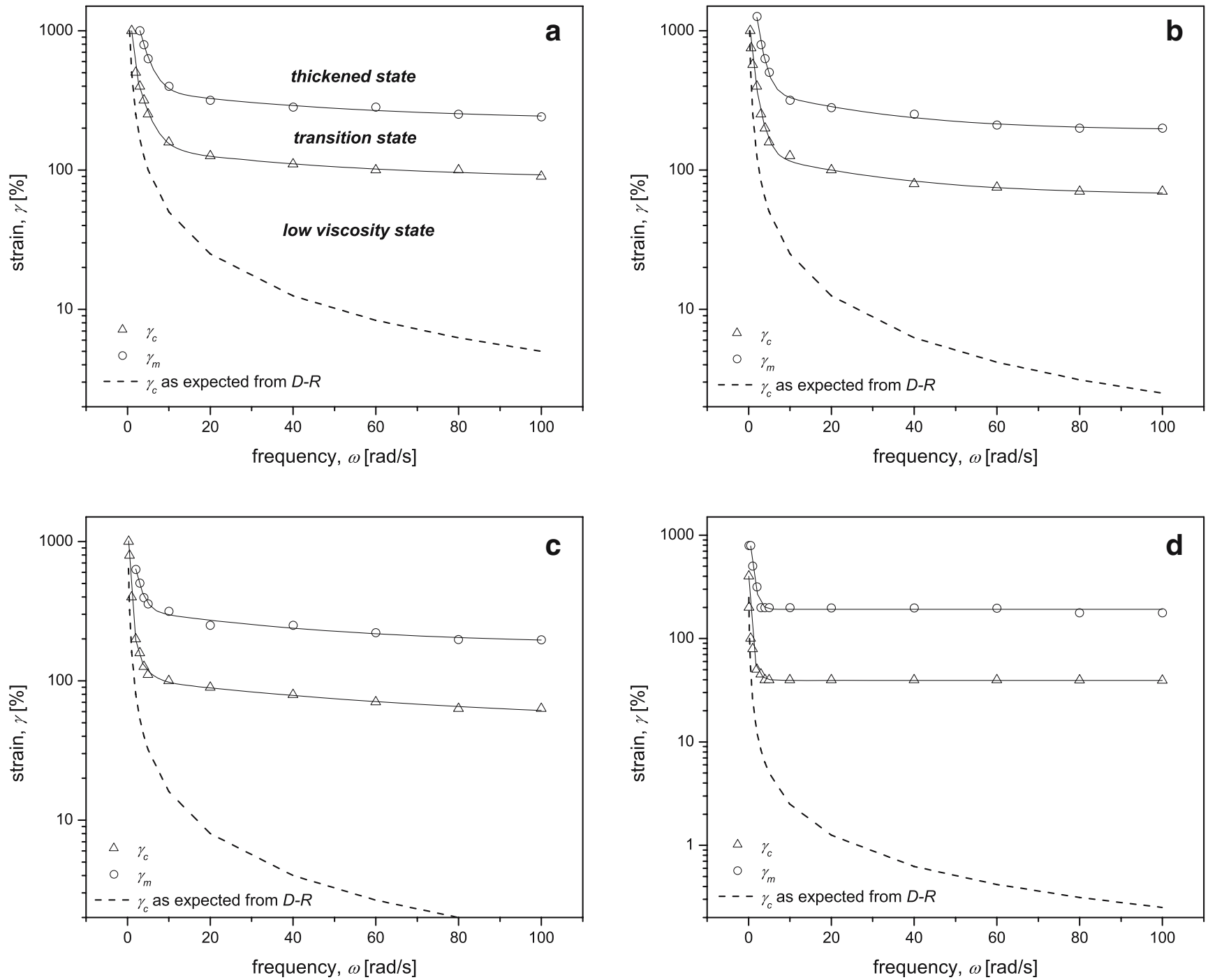

Fig. $7 \gamma_{\mathrm{c}}$ and $\gamma_{\mathrm{m}}$ as a function of $\omega$ for a $10 \% w / w, \mathbf{b} 12.5 \% \mathrm{w} / \mathrm{w}, \mathbf{c} 15 \% \mathrm{w} / \mathrm{w}$, and d $17.5 \% \mathrm{w} / \mathrm{w}$ silica-PPG suspensions. The hatched curves correspond to $\gamma_{c}$ predicted by applying the Delaware-Rutgers rule to steady-state data 
covering the plated surfaces with emery paper. This was confirmed by Walls et al. (2003) who investigated the influence of the tool surface roughness and showed that wall slip was substantially eliminated with rough tool geometries. Fourier transform (FT) rheology was nevertheless used in this study to confirm the otherwise lack of wall slip. FT rheology has established itself as a powerful tool for investigating rheological behavior in non-linear regimes of large amplitude oscillatory shear (LAOS), allowing the identification and quantification of higher harmonics. A detailed description of FT rheology and its applications has been given by Wilhelm (2002). In most cases, the Fourier analysis of the shear stress response indicates that nonlinearity is generally associated with the presence of odd harmonics of the fundamental excitation frequency when determining the shear stress response of viscoelastic fluids (Hyun et al. 2002; Wilhelm 2002). The presence of even harmonics, on the other hand, has been attributed to wall slip (Hatzikiriakos and Dealy 1992; Graham 1995; Yziquel et al. 1999). Recently, Atalik and Keunings (2004) have shown that wall slip is not a necessary condition for the presence of even harmonics in LAOS experiments, but the absence of even harmonics would still indicate wall slip not to occur.

The FT rheology results presented in this paper were obtained with the $15 \% \mathrm{w} / \mathrm{w}$ suspension, but are representative of all the concentrations. The dynamic stress response was recorded under three different sets of conditions corresponding to (a) the pre-transition state $\left(\gamma_{\mathrm{a}}=50 \%\right)$, (b) the transition state $\left(100 \%<\gamma_{b}<200 \%\right)$, and (c) the posttransition state $\left(\gamma_{\mathrm{a}}=400 \%\right)$, all measured at a constant $\omega$ of $10 \mathrm{rad} / \mathrm{s}(1.59 \mathrm{~Hz})$. These are illustrated for the dynamic strain sweep $(\omega=10 \mathrm{rad} / \mathrm{s})$ in Fig. 8. The harmonics

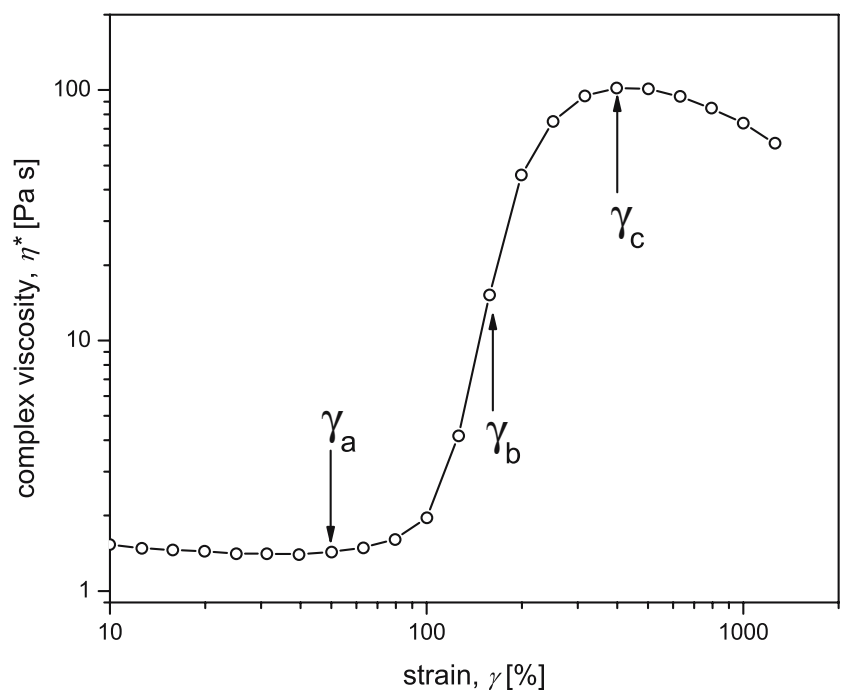

Fig. 8 Dynamic strain sweep for the $15 \% w / w$ silica-PPG suspension at a constant $\omega$ of $10 \mathrm{rad} / \mathrm{s}$, showing the testing conditions under which the Fourier transformations presented in Fig. 9 were obtained, i.e., at constant strains $\gamma_{\mathrm{a}}, \gamma_{\mathrm{b}}$, and $\gamma_{\mathrm{c}}$, respectively corresponding to the shear stress response under the different testing conditions are shown in Fig. 9 in the form of a FT spectrum. The intensity of the higher harmonics was normalized with respect to the intensity of the fundamental frequency, $\omega_{1}$. In addition to the third and fifth harmonics, which are typical of large strain amplitude experiments, a second harmonic appeared in the pretransition state (Fig. 9a), indicating possible wall slip at the tool-sample interface. The intensity of the second harmonic was nevertheless very low (less than $2 \%$ of the intensity of the first harmonic), and its influence on the measurements was negligible (Hyun et al. 2003). During the transition (Fig. 9b), the relative intensities of the third and fifth harmonics increased with respect to their values in the pre-transition state. This increase was consistent with the strongly non-linear nature of the strain-thickening transition.
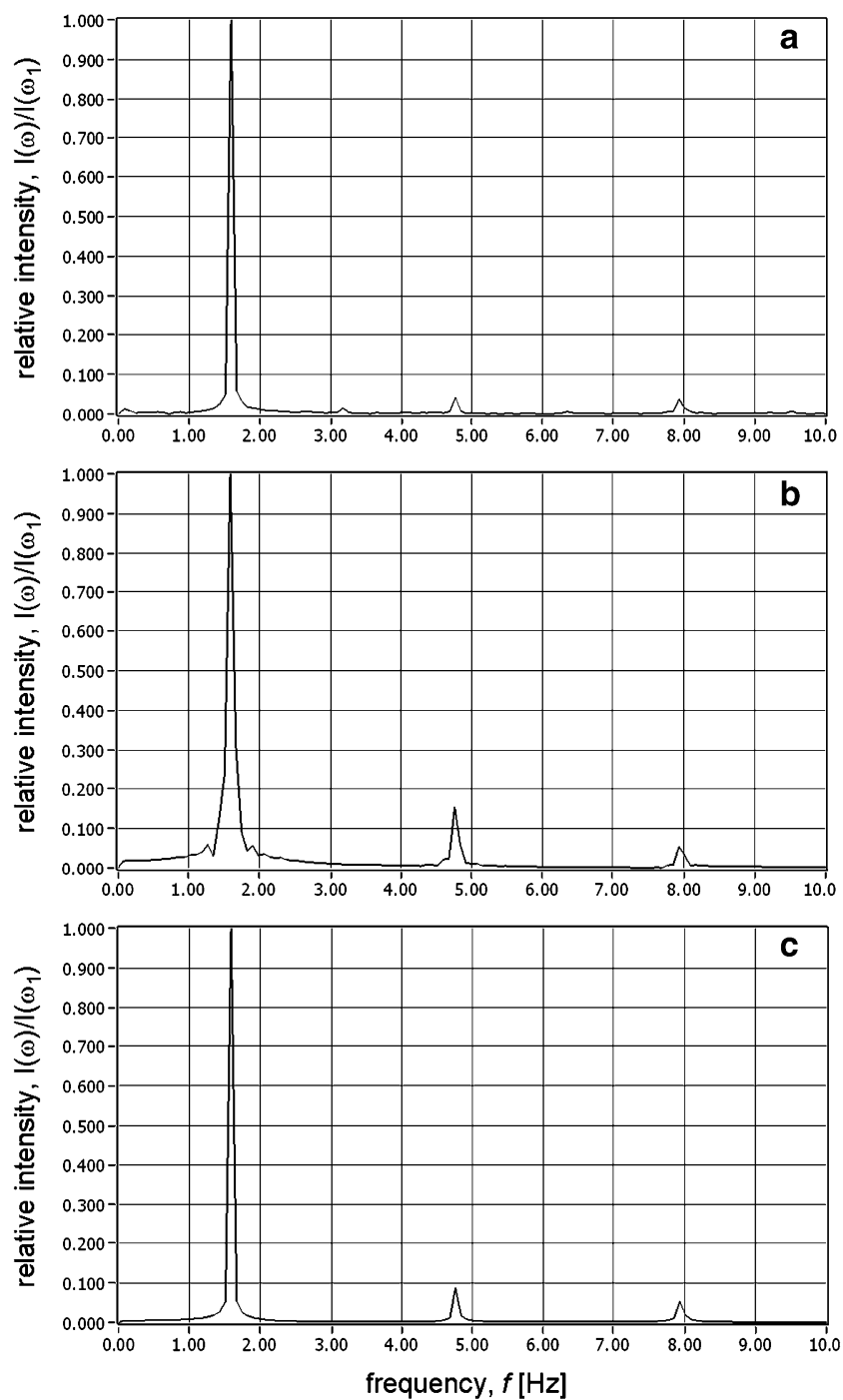

Fig. 9 Intensities of the harmonics normalized by the intensity of the fundamental excitation frequency reflecting the oscillatory sheared STF for $\mathbf{a}$ the pre-transition, $\mathbf{b}$ the transition, and $\mathbf{c}$ the post-transition state according to Fig. 8 
Even harmonics were not detected in the transition state, which indicates that wall slip did not occur. Finally, as seen in Fig. 9c, the non-linearity decreased once more in the posttransition state, i.e., the relative intensities of the higher harmonics were lower than those associated with the transition. No even harmonics were detected in this regime.

\section{Conclusion}

The pre- and post-transition viscosity of different STFs (colloidal suspensions of fused silica in polypropylene glycol) subjected to oscillating shear was found to be independent of the applied strain amplitude in frequency sweeps. Frequency sweeps corresponding to different set strain amplitudes showed a unique power law decrease of the viscosity with frequency, both below and above the shear-thickening transition. This decrease was more pronounced at frequencies above the transition, implying that shear thickening may disappear all together at sufficiently high frequencies. These trends were independent of the solids loading of the suspensions. Attempts to describe the thickening transitions using the Delaware-Rutgers rule were found only to give reasonable results at high strain amplitudes $(\gamma>300 \%)$. Similarly, for the present specimens, the critical shear stress obtained from investigations of the steady-state shearthickening behavior failed to account for the transition in the dynamic tests over the whole range of strain amplitudes. To provide a working description of the overall behavior for subsequent incorporation in structural models, the critical shear strains for the onset and the completion of the transition were plotted against frequency for the different concentrations so that the state of the suspension, i.e., whether it was in the pre-transition, transition, or post-transition state, could be determined for any arbitrary combination of strain and frequency. Fourier transform spectra of the shear stress response corresponding to the three states showed that nonlinearity associated with large strain amplitudes did not substantially influence the experimental results in this work and that wall slip did not occur, even at high strains.

Acknowledgment The authors gratefully acknowledge the Sports and Rehabilitation Engineering (SRE) program of the EPFL for the financial support, Prof. Norman J. Wagner and his group at the University of Delaware for numerous discussions of shear-thickening rheology, and Degussa for supplying the materials. They also acknowledge Lars E. Schmidt from LTC for fruitful discussions of FT rheology.

\section{References}

Atalik K, Keunings R (2004) On the occurrence of even harmonics in the shear stress response of viscoelastic fluids in large amplitude oscillatory shear. J Non-Newton Fluid 122:107-116
Barnes HA (1989) Shear-thickening (“dilatancy”) in suspensions of nonaggregating solid particles dispersed in Newtonian liquids. J Rheol 33:329-366

Bender J, Wagner NJ (1996) Reversible shear thickening in monodisperse and bidisperse colloidal dispersions. J Rheol 40:899-916

Boersma WH, Laven J, Stein HN (1990) Shear thickening (dilatancy) in concentrated dispersions. AIChE J 36:321-332

Boersma WH, Laven J, Stein HN (1992) Viscoelastic properties of concentrated shear-thickening dispersions. J Colloid Interface Sci 149:10-22

Bossis G, Brady JF (1989) The rheology of Brownian suspensions. J Chem Phys 91:1866-1874

Clarke B (1967) Rheology of coarse settling suspensions. Trans Inst Chem Eng 45:251-256

Doraiswamy D, Mujumdar AN, Tsao I, Beris AN, Danforth SC, Metzner AB (1991) The Cox-Merz rule extended - a rheological model for concentrated suspensions and other materials with a yield stress. J Rheol 35:647-685

Egres RG, Wagner NJ (2005) The rheology and microstructure of acicular precipitated calcium carbonate colloidal suspensions through the shear thickening transition. J Rheol 49:719-746

Fischer C, Braun SA, Bourban P-E, Michaud V, Plummer JG, Månson J-AE (2006a) Dynamic properties of sandwich structures with integrated shear-thickening fluids. Smart Mater Struct 15:14671475

Fischer C, Fauve M, Combaz E, Bourban P-E, Michaud V, Plummer JG, Rhyner H, Månson J-AE (2006b) Dynamic properties of materials for alpine skis. In: Moritz EF, Haake S (eds) The engineering of sports 6, vol. 1. Springer, Berlin Heidelberg New York, pp 263-268

Foss DR, Brady JF (2000) Structure, diffusion and rheology of Brownian suspensions by Stokesian dynamics simulation. J Fluid Mech 407:167-200

Graham MD (1995) Wall slip and the nonlinear dynamics of largeamplitude oscillatory shear flows. J Rheol 39:697-712

Hatzikiriakos SG, Dealy JM (1992) Role of slip and fracture in the oscillating flow of HDPE in a capillary. J Rheol 36:845-884

Helber R, Dockner F (1990) Vibration attenuation by passive stiffness switching mounts. J Sound Vib 138:47-57

Hoffmann RL (1972) Discontinuous and dilatant viscosity behavior in concentrated suspensions i. observation of a flow instability. J Colloid Interface Sci 16:155-173

Hoffmann RL (1974) Discontinuous and dilatant viscosity behavior in concentrated suspensions 2. Theory and experimental tests. J Colloid Interface Sci 46:491-496

Hyun K, Kim SH, Ahn KH, Lee SJ (2002) Large amplitude oscillatory shear as a way to classify the complex fluids. J Non-Newton Fluid 107:51-65

Hyun K, Nam JG, Wilhelm M, Ahn KH, Lee SJ (2003) Nonlinear response of complex fluids under LAOS (large amplitude oscillatory shear) flow. Korea-Aust Rheol J 15:97-105

Krishnamurthy LN, Wagner NJ, Mewis J (2005) Shear thickening in polymer stabilized colloidal dispersions. J Rheol 49:1347-1360

Laun HM, Bung R (1992) Rheological and small angel neutron scattering investigation of shear-induced particle structures of concentrated polymer dispersions submitted to plane Poiseuille and Couette flow. J Rheol 36:743-787

Laun HM, Bung R, Schmidt F (1991) Rheology of extremely shear thickening polymer dispersions. J Rheol 35:999-1034

Lee YS, Wagner NJ (2003) Dynamic properties of shear thickening colloidal suspensions. Rheol Acta 42:199-208

Lee YS, Wetzel ED, Wagner NJ (2003) The ballistic impact characteristics of Kevlar ${ }^{\circledR}$ woven fabrics impregnated with a colloidal shear thickening fluid. J Mater Sci 38:2825-2833

Maranzano BJ, Wagner NJ (2001a) The effects of interparticle interactions and particle size on reversible shear thickening: hard-sphere colloidal dispersions. J Rheol 45:1205-1222 
Maranzano BJ, Wagner NJ (2001b) The effects of particle size on reversible shear thickening of concentrated colloidal dispersions. J Chem Phys 114:10514-10527

Mewis J, Biebaut G (2001) Shear thickening in steady and superposition flows effect of particle interaction forces. J Rheol 40:9-16

Raghavan SR, Khan SA (1997) Shear-thickening response of fumed silica suspensions under steady and oscillatory shear. J Colloid Interface Sci 185:57-67
Schmidt LE, Leterrier Y, Vesin JM, Wilhelm M, Manson JAE (2005) Photorheology of fast UV-curing multifunctional acrylates. Macromol Mater Eng 290:1115-1124

Walls HJ, Caines SB, Sanchez AM, Khan SA (2003) Yield stress and wall slip phenomena in colloidal silica gels. J Rheol 47:847-868

Wilhelm M (2002) Fourier-transform rheology. Macromol Mater Eng 287:83-105

Yziquel F, Carreau PJ, Tanguy PA (1999) Non-linear viscoelastic behavior of fumed silica suspensions. Rheol Acta 38:14-25 